\title{
Novedades corológicas para cuatro especies de orquídeas en la comarca del Andévalo (Huelva, España)
}

Juan José Domínguez-Delgado \& Francisco Balao

Dpto. de Biología Vegetal y Ecología, Apdo. correos 1095, Avda. Reina Mercedes, Universidad de Sevilla, Sevilla, Spain

\section{Correspondencia:}

Francisco Balao

e-mail: fbalao@us.es

Recibido: 31 julio 2019

Aceptado: 25 febrero 2020

Publicado on-line: 20 abril 2020

Editado por: Marta Recio Criado

\section{Resumen}

Se constata por primera vez la presencia, en la comarca del Andévalo (Huelva), de varias especies de orquídeas: Ophrys bombyliflora Link, Orchis papilionacea L., Neotinea maculata (Desf.) Stearn y Cephalanthera longifolia (L.) Fritsch. Todo el material recolectado se encuentra depositado en el Herbario SEV de la Universidad de Sevilla.

Palabras clave: Cephalanthera, corología, Neotinea, Orchis, Ophrys

\begin{abstract}
Chorological novelties of four orchid species in the Andevalo area (Huelva, Spain)
\end{abstract}

The presence in the Andévalo region (Huelva) of several species of orchids is verified for the first time: Ophrys bombyliflora Link, Orchis papilionacea L., Neotinea maculata (Desf.) Stearn and Cephalanthera longifolia (L.) Fritsch. All the collected material is deposited in the SEV Herbarium of the University of Seville.

Keywords: Cephalanthera, chorology, Neotinea, Orchis, Ophrys
En este trabajo confirmamos la presencia de cuatro especies de orquídeas que hasta la fecha no se habían considerado para la flora del distrito andevalense del sector Marianico-Monchiquense en la provincia de Huelva (subprovincia LusoExtremadurense, Península lbérica; Rivas-Martínez, 2005).

Ophrys bombyliflora Link, Journ. Bot. (Scbrader) 1799 (2): 325 (1800)

ESPAÑA. - HUELVA: Zalamea la Real, dehesa, 325 $\mathrm{m}, \quad 37^{\circ} 41^{\prime} 18.3^{\prime \prime} \mathrm{N} \quad 6^{\circ} 40^{\prime} 43.5^{\prime \prime O}, \quad 01-\mathrm{IV}-2018$, J.J. Domínguez-Delgado (SEV 287215)

Especie de orquídea de pequeño porte, que crece entre claros de matorral y bosques aclarados, tanto en suelos ácidos como básicos, y cuyo labelo se asemeja a algunas especies de dípteros de la familia Bombyliidae.

La distribución conocida en la provincia de Huelva se limitaba a la zona del litoral (Silvestre, 1987; Sánchez Gullón et al., 2012). La población descubierta se estima en unos 30 individuos distribuidos en menos de $5 \mathrm{~m}^{2}$ y aparecen como acompañantes otras especies de orquídeas más comunes en el área (Ophrys apifera Huds., Ophrys tenthredinifera Willd., Orchis papilionacea L., Serapias lingua L. y Serapias parviflora Parl.).

Orchis papilionacea L., Syst. Nat. ed. 10, 2 : 1242 (1759)

ESPAÑA. - HUELVA: Zalamea la Real, dehesa, 325 $\mathrm{m}, \quad 37^{\circ} 41^{\prime} 18.3^{\prime \prime} \mathrm{N} \quad 6^{\circ} 40^{\prime} 43.5^{\prime \prime O}, \quad 31-I I I-2019, \quad J . J$. Domínguez-Delgado (SEV 288264)

Crece en prados y claros de matorral, generalmente sobre suelos ácidos. Posee una amplia distribución (Aedo, 2005), pero en Andalucía ha sido considerado como una especie poco frecuente (Silvestre, 1987), llegándose a excluir en las últimas revisiones del Andévalo y la Sierra Norte de Huelva (Valdés et al., 2008). Hasta la fecha, se ha encontrado un único ejemplar en la localización mencionada.

Neotinea maculata (Desf.) Stearn in Ann. Mus. Goulandris 2: 79 (1975)

ESPAÑA. - HUELVA: Minas de Riotinto, pinar, $259 \mathrm{~m}$, $37^{\circ} 40^{\prime} 16.5^{\prime \prime} \mathrm{N} \quad 6^{\circ} 33^{\prime} 45.6^{\prime \prime O}, \quad 31-I I I-2019, \quad J . J$. Domínguez-Delgado (SEV 288263) 
Orquídea que se caracteriza por presentar un moteado (más o menos intenso en función del individuo) de color purpúreo tanto en las hojas como en las flores. Aunque se conoce su presencia en algunos enclaves de la provincia de Huelva tanto en la Sierra de Aracena como en la zona del litoral (Rivera \& Cabezudo, 1985; Silvestre, 1987; López,
2005), en las últimas revisiones florísticas no se contempla dentro del distrito andevalense (Valdés et al., 2008). Se constata la presencia en varios enclaves dispersos asociados a pinares en la Cuenca Minera con densidades poblacionales bajas (Figura 1).

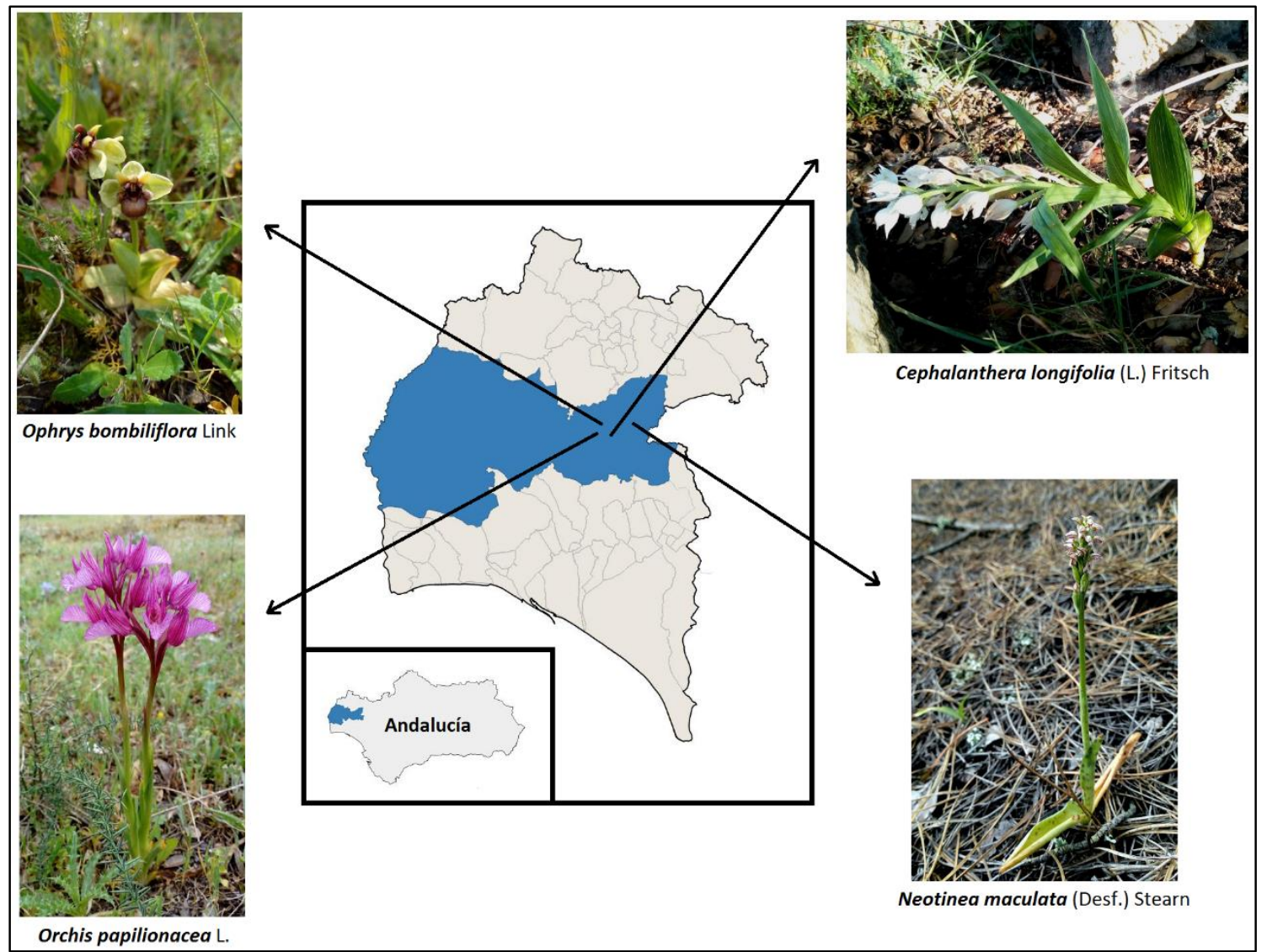

Figura 1. Localización de los taxones de orquídeas encontrados en el Andévalo. Autor: JJ Domínguez-Delgado

Figure 1. Localities of the orchids found in the Andevalo area. Author: JJ Domínguez-Delgado

Cephalanthera longifolia (L.) Fritsch in Oester. Bot. Z. 38: 81 (1888)

ESPAÑA. - HUELVA: Zalamea la Real, dehesa, 335 $\mathrm{m}, \quad 37^{\circ} 41^{\prime} 4.5^{\prime \prime} \mathrm{N} \quad 6^{\circ} 40^{\prime} 46.9^{\prime \prime O}, \quad 13-$ IV-2019, J.J. Domínguez-Delgado (SEV 288265)

Especie que posee un corto rizoma subterráneo en lugar de tubérculo desarrollados y cuya inflorescencia suele contener 10-15 flores (de color blanco). Suele aparecer en dehesas y bosques de caducifolios.

Se conoce su presencia dentro de la provincia de Huelva (Alarcón \& Aedo, 2005), donde aparece con cierta frecuencia en la Sierra de Aracena (Rivera \& Cabezudo, 1985). La población formada por un único ejemplar (Figura 1) constata su presencia en el distrito andevalense, hasta ahora excluido de su distribución (Valdés et al., 2008).

\section{Agradecimientos}

A Félix Lancha Gómez, gran conocedor de la flora zalameña y de sus alrededores, por reportar la existencia de la mayoría de los taxones mencionados. Al Servicio General de Investigación de Herbario de la Universidad de Sevilla, en cuyas instalaciones se ha preparado y se almacena actualmente el material de estudio.

\section{Bibliografía}

Aedo, C. (2005). Orchis. In S. Castroviejo et al. (Eds.), Flora lberica, 21 (pp. 115-145), Madrid: CSIC.

Alarcón, M. L. \& Aedo, C. (2005). Cephalanthera. In S. Castroviejo et al. (Eds.), Flora Iberica, 21 (pp. 55-57), Madrid: CSIC. 
López, N. (2005). Neotinea. In S. Castroviejo et al. (Eds.), Flora Iberica, 21 (pp. 112-114), Madrid: CSIC.

Rivas-Martínez, S. (2005). Avances en Geobotánica. Discurso de Apertura del Curso Académico de la Real Academia Nacional de Farmacia del año 2005.

http://www.globalbioclimatics.org/book/ranf2005. pdf. Consulta realizada en julio de 2019.

Rivera, J. \& Cabezudo, B. (1985). Aportaciones al conocimiento florístico de la Sierra de Aracena (Huelva, España). Acta Botanica Malacitana, 10, 61-78.
Sánchez Gullón, E., Nieva, F. J. J., de las Heras, M. A. \& Muñoz, A. (2012). Notas florísticas para el litoral de Huelva y provincia. V. Lagascalia, 32, 318-319.

Silvestre, S. (1987). Orchidaceae. In Valdés, B., Talavera, S. \& Fernández-Galiano, E. (Eds.), Flora Vascular de Andalucía Occidental, 3 (pp. 495-519). Barcelona: Ketres.

Valdés, B., Santa-Bárbara, C., Vicent, C. \& Muñoz, A. (2008). Catálogo florístico del Andévalo y Sierra de Huelva (plantas vasculares). Lagascalia, 28, 117-409. 